\title{
A New Scoring System to Predict Poor Clinical Outcomes in Acute Nonvariceal Upper Gastrointestinal Bleeding Patients with High-Risk Stigmata
}

\author{
Zhiyu Dong $\mathbb{D}^{D}$, Junwen Wang, Tingting Zhan, Haiqin Zhang, Lisha Yi, and Shuchang Xu \\ Department of Gastroenterology, Tongji Hospital, Tongji University School of Medicine, Shanghai, China \\ Correspondence should be addressed to Shuchang Xu; xschang@163.com
}

Received 31 October 2017; Revised 12 January 2018; Accepted 15 January 2018; Published 12 March 2018

Academic Editor: Cristiano Pagnini

Copyright (C) 2018 Zhiyu Dong et al. This is an open access article distributed under the Creative Commons Attribution License, which permits unrestricted use, distribution, and reproduction in any medium, provided the original work is properly cited.

\begin{abstract}
Aims. To explore the risk factors for rebleeding in acute nonvariceal upper gastrointestinal bleeding patients with high-risk stigmata after endoscopic hemostasis and to develop a new scoring system for them. Methods. A retrospective single-center study was conducted from January 2012 to June 2017. The logistic regression model was used to explore risk factors of poor clinical outcomes. Accuracy of new scoring systems was compared with Rockall score (RS) and Glasgow-Blatchford score (GBS) using receiver operating characteristics curve. Results. Two hundred nine patients were included. In multivariate regression analysis, systolic blood pressure, endoscopic hemostasis method, hemoglobin, blood urea nitrogen, and serum creatinine were identified as indicators for rebleeding. New scoring systems with 4 variables and 5 variables based on these 5 risk factors were chosen. The 4-variable scoring system outperformed GBS in predicting rebleeding while 5-variable scoring system outperformed RS and GBS in predicting rebleeding significantly. Score 2 was identified as the best cut-off of these 2 scoring systems. Conclusions. Systolic blood pressure, endoscopic hemostasis method, hemoglobin, blood urea nitrogen, and serum creatinine were all associated with poor clinical outcomes. The new scoring systems had greater accuracy than RS and GBS in predicting rebleeding. Further external validation should be performed to verify the results.
\end{abstract}

\section{Introduction}

Acute upper gastrointestinal bleeding (AUGIB) is a common disease with an incidence of 100 to 180 per 100,000 adults [1] while acute nonvariceal upper gastrointestinal bleeding (ANVUGIB) accounted for $80 \%-90 \%$ of AUGIB [2]. A systemic review showed that the incidence of rebleeding within 7 days was $13.9 \%$ while incidence of mortality was $8.6 \%$ in ANVUGIB patients [3]. Endoscopy plays an important role in diagnosis and treatment in ANVUGIB. Most ANVUGIB patients in European accepted endoscopy within 24 hours, which was considered important in management of ANVUGIB patients [4]. The endoscopic manifestation was also identified as key indicator for management of ANVUGIB [5]. Thanks to the development of endoscopic hemostasis and an increase in medical resource, the endoscopic hemostasis could be applied to the early stage of ANVUGIB once the high-risk stigmata have been found. This standpoint was recommended in many guidelines and international consensus [6-9]. Thus, the early assessment and precise management of ANVUGIB patients with high-risk stigmata after endoscopic hemostasis were also crucial.

Several scoring systems for ANVUGIB have been developed to assess the risk of patients. Rockall score (RS) is the first established and validated scoring system [10]. The system consists of multiple clinical data and endoscopic manifestation. Glasgow-Blatchford score (GBS) is the second validated scoring system [11]. The system includes UGIB symptoms and clinical data. Recently, many guidelines and international consensus recommended the prognostic scoring systems could be used for early classification of UGIB patients [6-9]. However, whether these scoring systems were suitable for the high-risk stigmata patients after endoscopic hemostasis was barely studied.

The aim of this study was to explore the risk factors for rebleeding in ANVUGIB patients with high-risk stigmata 
after endoscopic hemostasis. Then, we aimed to develop new scoring systems for them and compared the new systems with existing systems retrospectively.

\section{Methods}

Data were collected from consecutive patients found to have ANVUGIB with high-risk stigmata and received endoscopic hemostasis over a 5-year period, from January 2012 to June 2017, who were referred to Tongji Hospital in Shanghai, China. The ANVUGIB with high-risk stigmata was defined as spurting, gushing, oozing bleeding or nonbleeding visible vessel in the cases of peptic ulcers and spurting, gushing bleeding or nonbleeding visible vessel in the cases of other diseases.

This was a retrospective study including all medical records. Admission history, clinical and laboratory data, endoscopic manifestation, endoscopic hemostasis methods, and clinical outcomes were recorded. All patients were treated with standard supportive treatment, fluid resuscitation, and high-dose acid suppression, $80 \mathrm{mg}$ pantoprazole (iv), after endoscopic hemostasis.

2.1. Data Collection. The following data were collected for each patient: demographic data, UGIB symptoms, history of UGIB and drug use, vital signs, laboratory results, endoscopic manifestation, endoscopic diagnosis, endoscopic hemostasis methods, and clinical outcome. The RS and GBS were calculated using the collected data for each patient.

Among them, laboratory results consist of hemoglobin (HB), blood urea nitrogen (BUN), serum creatinine (Scr), aspartate transaminase (AST), alanine aminotransferase (ALT), and blood glucose.

2.2. Endoscopic Procedure. All endoscopic procedures were performed by experienced endoscopist who had experience in endoscopic examination and hemostasis. Endoscopic diagnosis of bleeding was classified into spurting hemorrhage, oozing hemorrhage, and visible vessel. As for peptic ulcer bleeding, the diagnosis was followed as Forrest classification. Endoscopic hemostasis consisted of monotherapy and multiple therapies combined. The monotherapy included thermal hemostasis using argon and mechanical hemostasis using titanium clip while multiple therapies combined was defined as submucosal epinephrine injection plus thermal or mechanical hemostasis. The endoscopic hemostasis methods were chosen based on the endoscopists' judgement.

2.3. Clinical Outcome. The clinical outcome of the current study was defined as rebleeding after endoscopic hemostasis. Rebleeding was defined as one or more signs of bleeding after primary bleeding stopped, including fresh hematemesis or hematochezia, melena with instable vital signs, or reduction in hemoglobin levels by $3 \mathrm{~g} / \mathrm{dL}$ or more.

2.4. Statistical Analysis. All analyses were performed using $\mathrm{R}$ language version 3.1.1(R Foundation for Statistical Computing, Vienna, Austria) and MedCalc version 11.4.2.0 (MedCalc software, http://www.medcalc.be).
To explore the risk of poor clinical outcomes for clinical data, the factors whose $P<0.1$ in univariate regression analysis were included while multivariate logistic regression analysis using stepwise selection to achieve the lowest Akaike information criterion (AIC) was used to identify independent indicators of the poor clinical outcomes. Continuous variables were converted into ordinal categorical variables based on quartile.

Then, the continuous variables were also converted into unordered categorical variables based on quartile in order to determine the risk of poor clinical outcomes for each quartile of laboratory data. The binary logistic regression analysis was applied with the lowest quartiles as reference. Model 1 was unadjusted. Model 2 was adjusted for risk factors obtained in final multivariate model for each clinical outcome.

The scoring systems were made using all combinations of risk factors obtained. The cut-off of each laboratory data was identified using the intersection of the best 5 cut-offs for each clinical outcome. The best cut-off was based on Youden's index.

The accuracy of the scoring systems to predict clinical outcomes was evaluated by receiver operating characteristics curves (ROC) with 95\% confidence intervals. The areas under ROC curves (AUC) were compared using chi-square tests according to the method described by Delong et al. [12].

All reported $P$ values were two-sided with $P<0.05$ defined as statistical significance.

\section{Results}

3.1. Patient Characteristics. A total of 209 patients were included in the study. Table 1 describes the demographics and clinical and endoscopic characteristics of these patients. One hundred seventy-three males and 36 females were studied, whose median age was 58 . For the clinical outcomes, 38 patients bled one or more times after primary bleeding stopped and 44 patients had requirement of blood transfusion. Duodenal ulcer and gastric ulcer were the main causes of ANVUGIB. For endoscopic hemostasis, 172 patients accepted monotherapy consisting of 101 mechanical hemostasis and 71 thermal hemostasis. Thirty-seven patients received multiple hemostasis combined.

3.2. Logistic Regression Analysis for Rebleeding. Table 2 demonstrates the differences of risk factors between the rebleeding group and no rebleeding group by giving statistical significance using logistic regression model. In multivariate regression analysis, systolic blood pressure (SBP) (<90 mmHg), endoscopic hemostasis method, HB, BUN, and Scr were included in final regression model. But only SBP, endoscopic hemostasis method, HB, and Scr were the independent indicators for rebleeding.

As demonstrated in Table 3, the results of logistic regression analysis revealed the risk of $\mathrm{HB}, \mathrm{BUN}$, and Scr, which were considered as unordered categorical variables. After adjusted for SBP, endoscopic hemostasis method, and other included laboratory data, the results showed increased risk of rebleeding with $\mathrm{HB}$ and $\mathrm{Scr}$ ascending. The $\mathrm{Q} 4$ of $\mathrm{HB}$ 
TABLE 1: The characteristics of acute nonvariceal upper gastrointestinal bleeding patients enrolled.

\begin{tabular}{|c|c|}
\hline & Number of patients \\
\hline \multicolumn{2}{|l|}{ Clinical characteristics } \\
\hline Age: $\operatorname{median}(\mathrm{IQR})^{\mathrm{a}}$ & $58(42,66)$ \\
\hline \multicolumn{2}{|l|}{ Gender } \\
\hline Male & 173 \\
\hline Female & 36 \\
\hline \multicolumn{2}{|l|}{ UGIB symptom $(s)^{b}$} \\
\hline Melena & 181 \\
\hline Hematemesis & 107 \\
\hline Syncope & 23 \\
\hline \multicolumn{2}{|l|}{ Outcomes } \\
\hline Rebleeding & 38 \\
\hline Blood transfusion & 44 \\
\hline Further intervention & 15 \\
\hline Mortality & 9 \\
\hline \multicolumn{2}{|l|}{ Endoscopic characteristics } \\
\hline \multicolumn{2}{|l|}{ Manifestation } \\
\hline Gastric ulcer & 57 \\
\hline Duodenal ulcer & 101 \\
\hline Anastamotic ulcer & 3 \\
\hline Esophageal ulcer & 3 \\
\hline Gastritis & 5 \\
\hline Duodenitis & 10 \\
\hline Anastamotic bleeding & 10 \\
\hline Mallory-Weiss & 16 \\
\hline Gastric vascular ectasia & 1 \\
\hline Malignancy & 3 \\
\hline \multicolumn{2}{|l|}{ Endoscopic hemostasis } \\
\hline Monotherapy & 172 \\
\hline Multiple therapies combined & 37 \\
\hline
\end{tabular}

${ }^{\mathrm{a}} \mathrm{IQR}$ : interquartile range. ${ }^{\mathrm{b}}$ Some patients presented with more than one symptom.

and Scr was significantly associated with higher hazard compared to those in the lowest quartile. The Q3 of BUN has the highest hazard in the 4 quartiles. It was inverted U-shaped relation between BUN and risk of rebleeding. Moreover, there is almost no difference between the hazard of the Q2 (range $6.6 \sim 10 \mathrm{mmol} / \mathrm{L}$ ) and Q1 (range $<6.6 \mathrm{mmol} / \mathrm{L}$ ). Despite no statistically significant, the Q3 and Q4 have higher hazard compared to Q1 and Q2 in multivariate analysis while the Q4 of BUN was significantly associated with higher hazard compared to the lowest quartile in univariate analysis. Thus, HB, BUN, and Scr were all the key predictors for rebleeding.

3.3. Development of the New Scoring Systems. Totally, 5 factors were included in our new scoring systems based on the final multivariate regression model. The cut-off of continuous variables including $\mathrm{HB}, \mathrm{BUN}$, and Scr was identified using the intersection of the best 5 cut-offs based on Youden's index for each clinical outcome (Table 4). In order to make the cut-off well remembered and meet the clinical practicality, the cut-offs of BUN and Scr were set as $9.5 \mathrm{mmol} / \mathrm{L}$ and $100 \mu \mathrm{mol} / \mathrm{L}$, respectively. As for $\mathrm{HB}$, the best 5 cut-offs for rebleeding were around $75 \mathrm{~g} / \mathrm{L}$ or $85 \mathrm{~g} / \mathrm{L}$. Calculating the sensitivity and specificity of both cut-offs, the results showed too low sensitivity in cut-off $75 \mathrm{~g} / \mathrm{L}$ (rebleeding sensitivity/specificity $0.47 / 0.86$ ) and cut-off $85 \mathrm{~g} / \mathrm{L}$ has better result (rebleeding sensitivity/specificity $0.58 / 0.75$ ). Thus, $\mathrm{HB}<85 \mathrm{~g} / \mathrm{L}$ was finally selected as cut-off value. All of these 5 factors were weighted equally for simplicity.

A total of 31 combinations were created and AUC of each was calculated. Among them, the set of variables (from 1 to 5 ) that yielded the highest AUC value is shown in Table 5. Four-variable (Scr $>100 \mu \mathrm{mol} / \mathrm{L}$; BUN $>9.5 \mathrm{mmol} / \mathrm{L} ; \quad \mathrm{HB}<85 \mathrm{~g} / \mathrm{L} ;$ monotherapy) and 5variable $(\mathrm{Scr}>100 \mu \mathrm{mol} / \mathrm{L} ; \mathrm{BUN}>9.5 \mathrm{mmol} / \mathrm{L} ; \mathrm{HB}<85 \mathrm{~g} / \mathrm{L}$; monotherapy; $\mathrm{SBP}<90 \mathrm{mmHg}$ ) scoring systems were the best score for predicting rebleeding. The cut-off 2 in 4variable scoring system provided the high sensitivity (rebleeding 94.7\%) and patients with score 0 had no risk for rebleeding. The cut-off 2 in 5 -variable scoring system provided high sensitivity (94.7\%) while patients with score 0 had no risk for rebleeding either.

3.4. Comparison of New Scoring Systems with RS and GBS. In predicting rebleeding, 4-variable scoring system (AUC 0.78 $(0.71-0.85))$ performed as well as RS (AUC $0.70(0.61-0.79)$ ) and outperformed GBS (AUC $0.71(0.62-0.8)$ ) significantly $(P=0.049)$ while 5 -variable scoring system (AUC 0.79 $(0.72-0.86))$ outperformed both RS $(P=0.046)$ and GBS $(P=0.021)$ significantly (Table 6).

\section{Discussion}

ANVUGIB is a common digestive system disease and a frequent cause of poor clinical outcome. Recently, international consensus recommended the prognostic scoring systems should be used for early assessment of ANVUGIB patients [6-9]. Early assessment of patients at high risk can improve the efficiency of treatment and clinical outcomes for patients. For example, better allocation of medical resources could be administered after early identification of patients. A systemic review evaluating the accuracy of RS and GBS demonstrated that GBS excelled RS in identifying patients who did not require any intervention [13]. Recently, a multicenter prospective cohort study enrolling 1584 AUGIB patients illustrated the value of GBS in predicting hospital-based intervention and the superiority of RS in predicting death [14]. As the development of endoscopy, more patients could accept endoscopy within 24 hours and the endoscopic manifestation was considered as a key indicator for management. The endoscopic hemostasis was superior to pharmacotherapy in patients with high-risk stigmata [15]. Thus, the patients with high-risk stigmata found in endoscopy could receive hemostasis simultaneously. The improved clinical pathway decreased the risk of these patients, but the existing scoring systems were not updated to fit changed medical condition and whether the existing scoring systems are suitable for high-risk stigmata patients after endoscopic 
TABLE 2: Univariate and multivariate analysis between rebleeding case and no rebleeding case.

\begin{tabular}{|c|c|c|c|c|}
\hline & Rebleeding case $(n=38)$ & No rebleeding case $(n=171)$ & $P 1$ value & $P 2$ value \\
\hline Age $(>60)$, number $(\%)$ & $17(44.7 \%)$ & $66(38.6 \%)$ & 0.485 & \\
\hline Sex (male), number (\%) & $30(78.9 \%)$ & $143(83.6 \%)$ & 0.491 & \\
\hline HR (>100 beats/min), number (\%) & $12(31.6 \%)$ & $27(15.8 \%)$ & 0.027 & \\
\hline SBP (<90 mmHg), number (\%) & $5(13.2 \%)$ & $6(3.5 \%)$ & 0.025 & 0.016 \\
\hline Alcohol (yes), number (\%) & $9(23.7 \%)$ & $31(18.1 \%)$ & 0.433 & \\
\hline Smoke (yes), number (\%) & $14(36.8 \%)$ & $50(29.2 \%)$ & 0.359 & \\
\hline UGIB history (yes), number (\%) & $8(21.1 \%)$ & $40(23.4 \%)$ & 0.757 & \\
\hline Multiple therapies, number (\%) & $3(7.9 \%)$ & $34(19.9 \%)$ & 0.092 & 0.015 \\
\hline HR/SBP, mean (SD) & $0.89(0.38)$ & $0.73(0.21)$ & 0.023 & \\
\hline $\mathrm{HB}$, mean $(\mathrm{SD}), \mathrm{g} / \mathrm{L}$ & $86.82(26.94)$ & $105.25(26.47)$ & 0.001 & 0.002 \\
\hline BUN, mean (SD), mmol/L & $13.53(6.92)$ & $10.55(5.77)$ & 0.004 & 0.115 \\
\hline Creatinine, mean $(\mathrm{SD}), \mu \mathrm{mol} / \mathrm{L}$ & $103.55(46.49)$ & $82.08(49.39)$ & 0.001 & 0.004 \\
\hline Glucose, mean (SD), mmol/L & $8.09(3.44)$ & $7.93(3.97)$ & 0.188 & \\
\hline ALT, mean (SD), U/L & $41.13(70.54)$ & $28.71(22.28)$ & 0.268 & \\
\hline AST, mean (SD), U/L & $42.37(84.1)$ & $24.73(26.97)$ & 0.693 & \\
\hline
\end{tabular}

HR: heart rate; SBP: systolic blood pressure; HB: hemoglobin; BUN: blood urea nitrogen; AST: aspartate transaminase; ALT: alanine aminotransferase; multiple therapies was defined as submucosal epinephrine injection plus thermal or mechanical hemostasis.

TABLE 3: The risk of rebleeding for each quartile of laboratory data.

\begin{tabular}{|c|c|c|c|c|c|}
\hline & Q1 & Q2 & Q3 & Q4 & $P$ for trend \\
\hline $\mathrm{HB}(\mathrm{g} / \mathrm{L})$ & $n=52$ & $n=52$ & $n=49$ & $n=56$ & \\
\hline Range & $121 \sim 160$ & $103 \sim 120$ & $81 \sim 102$ & $42 \sim 80$ & \\
\hline Model 1 & Ref. & $1.87(0.53-7.53)$ & $2.34(0.69-9.3)$ & $6.16(2.11-22.65)$ & \\
\hline$P$ values & & 0.344 & 0.189 & 0.002 & 0.001 \\
\hline Model 2 & Ref. & $1.42(0.35-6.19)$ & $2.55(0.71-10.51)$ & $5.6(1.73-22.2)$ & \\
\hline$P$ values & & 0.623 & 0.163 & 0.007 & 0.002 \\
\hline $\mathrm{BUN}(\mathrm{mmol} / \mathrm{L})$ & $n=53$ & $n=54$ & $n=53$ & $n=49$ & \\
\hline Range & $2.85 \sim 6.5$ & $6.6 \sim 10$ & $10.1 \sim 14.7$ & $14.8 \sim 42.8$ & \\
\hline Model 1 & Ref. & $1.43(0.43-5.13)$ & $2.51(0.84-8.52)$ & $4.24(1.48-14.04)$ & \\
\hline$P$ values & & 0.564 & 0.111 & 0.01 & 0.004 \\
\hline Model 2 & Ref. & $1.08(0.29-4.2)$ & $2.56(0.77-9.54)$ & $2.08(0.62-7.74)$ & \\
\hline$P$ values & & 0.911 & 0.136 & 0.246 & 0.115 \\
\hline $\operatorname{Scr}(\mu \mathrm{mol} / \mathrm{L})$ & $n=55$ & $n=55$ & $n=48$ & $n=51$ & \\
\hline Range & $37 \sim 66$ & $67 \sim 76$ & $77 \sim 93$ & $94 \sim 669$ & \\
\hline Model 1 & Ref. & $1.7(0.53-5.98)$ & $1.71(0.51-6.15)$ & $5.45(1.96-17.83)$ & \\
\hline$P$ values & & 0.379 & 0.39 & 0.002 & 0.001 \\
\hline Model 2 & Ref. & $1.83(0.53-6.95)$ & $1.97(0.54-7.76)$ & $5.94(1.86-22.13)$ & \\
\hline$P$ values & & 0.351 & 0.31 & 0.004 & 0.004 \\
\hline
\end{tabular}

HB: hemoglobin; BUN: blood urea nitrogen; Scr: serum creatinine; Model 1: crude, no adjustment; Model 2: adjusting for risk factors in final multivariate model; Ref: reference.

hemostasis was barely studied. Thus, it is necessary to construct new scoring systems with higher accuracy and better performance for ANVUGIB patients with high-risk stigmata after endoscopic hemostasis.

To make the cut-off well remembered and meet the clinical practicality, the cut-off of $\mathrm{HB}, \mathrm{BUN}$, and Scr was set as $85 \mathrm{~g} / \mathrm{L}, 9.5 \mathrm{mmol} / \mathrm{L}$, and $100 \mu \mathrm{mol} / \mathrm{L}$. HB, BUN, and Scr were all considered as crucial indicators for prognosis. Among them, HB and BUN were included in GBS, but the complexity of calculating made doctors seldom used this risk score. Additionally, there was not obvious difference between BUN (6.6 10 mmol/L) group and BUN $(\leq 6.5 \mathrm{mmol} / \mathrm{L})$ group in predicting rebleeding, but BUN $(>10 \mathrm{mmol} / \mathrm{L})$ group had increased hazard of rebleeding. Moreover, Scr was not included in GBS, but it is really a key indicator for poor clinical outcomes. As an ordinal categorical variable, Scr 
TABLE 4: The best 5 cut-offs of each laboratory data for predicting rebleeding.

\begin{tabular}{lccccc}
\hline & 5 th & 4 th & 3rd & 2nd & 1 st \\
\hline HB $(\mathrm{g} / \mathrm{L})$ & 74 & 84 & 77 & 86 & 73 \\
Youden's index & 0.333 & 0.333 & 0.336 & 0.342 & 0.345 \\
BUN (mmol/L) & 11.9 & 9.7 & 9.3 & 9.8 & 9.4 \\
Youden's index & 0.254 & 0.257 & 0.260 & 0.263 & 0.272 \\
Scr $(\mu \mathrm{mol} / \mathrm{L})$ & 94 & 95 & 99 & 100 & 102 \\
Youden's index & 0.281 & 0.287 & 0.287 & 0.292 & 0.295 \\
\hline
\end{tabular}

HB: hemoglobin; BUN: blood urea nitrogen; Scr: serum creatinine; Youden's index: sensitivity + specificity -1 .

TABLE 5: The combination of variables that yielded the highest AUC value with sensitivity and specificity.

\begin{tabular}{lccccc}
\hline $\begin{array}{l}\text { Number of } \\
\text { variable }\end{array}$ & $\begin{array}{c}\text { Variables for } \\
\text { rebleeding }\end{array}$ & AUC & Cut-off & Sensitivity & Specificity \\
\hline 1 & $\mathrm{HB}<85 \mathrm{~g} / \mathrm{L}$ & 0.67 & 1 & 57.9 & 75.4 \\
2 & $\mathrm{Scr}>100 \mu \mathrm{mol} / \mathrm{L}$ & 0.74 & 1 & 76.3 & 71.3 \\
& $\mathrm{HB}<85 \mathrm{~g} / \mathrm{L}$ & & 2 & 21.1 & 94.2 \\
3 & $\mathrm{Scr}>100 \mu \mathrm{mol} / \mathrm{L}$ & 0.76 & 1 & 100 & 10.5 \\
& $\mathrm{HB}<85 \mathrm{~g} / \mathrm{L}$ & & 2 & 71.1 & 77.8 \\
& $\mathrm{Monotherapy}$ & & 3 & 18.4 & 95.3 \\
4 & $\mathrm{Scr}>100 \mu \mathrm{mol} / \mathrm{L}$ & 0.78 & 1 & 100 & 7.6 \\
& $\mathrm{BUN}>9.5 \mathrm{mmol} / \mathrm{L}$ & & 2 & 94.7 & 43.9 \\
& $\mathrm{HB}<85 \mathrm{~g} / \mathrm{L}$ & & 3 & 52.6 & 88.3 \\
& $\mathrm{Monotherapy}$ & & 4 & 15.8 & 95.3 \\
5 & $\mathrm{Scr}>100 \mu \mathrm{mol} / \mathrm{L}$ & 0.79 & 1 & 100 & 7.6 \\
& $\mathrm{BUN}>9.5 \mathrm{mmol} / \mathrm{L}$ & & 2 & 94.7 & 42.7 \\
& $\mathrm{HB}<85 \mathrm{~g} / \mathrm{L}$ & & 3 & 57.9 & 86.0 \\
& $\mathrm{Monotherapy}$ & & 4 & 23.7 & 95.3 \\
& $\mathrm{SBP}<90 \mathrm{mmHg}$ & & 5 & - & - \\
\hline
\end{tabular}

HB: hemoglobin; BUN: blood urea nitrogen; Scr: serum creatinine; SBP: systolic blood pressure; monotherapy was defined as mechanical or thermal hemostasis.

was independent predictor for rebleeding in multivariate regression. As an unordered categorical variable, Scr $(\geq 94 \mu \mathrm{mol} / \mathrm{L})$ group had several risk of poor clinical outcomes compared with Scr $(<94 \mu \mathrm{mol} / \mathrm{L})$ group. After integrated analysis of logistic regression model and the best 5 cut-offs of each laboratory index, we adjusted the cut-off of these 3 laboratory data to most fit and easy-remembered status, which were different from the cut-off in GBS. SBPs were also indicators of patients' condition. A previous trial demonstrated that the shock index, HR/SBP, was independent predictor for high-risk stigmata and endoscopic intervention and the simple score consisting of shock index, BUN/Scr and "no daily use PPI one week before examination", was superior to GBS [16]. However, the shock index was not included in final multivariate regression model for rebleeding while SBPs were independent predictors for rebleeding in our results. Then, the frequently used cut-off "SBP $<90$ mmHg" was set. Recently, the international consensus and guidelines demonstrated the mechanical, thermal
TABLE 6: Accuracy of new scoring systems compared with existing scoring systems.

\begin{tabular}{lccc}
\hline & $\begin{array}{c}\text { Area under the } \\
\text { receiver-operator } \\
\text { curves (AUC) }\end{array}$ & $\begin{array}{c}95 \% \\
\text { confidence } \\
\text { interval }\end{array}$ & $\begin{array}{c}\text { Comparison } \\
\text { to new score } \\
(P \text { value })\end{array}$ \\
\hline 4 variables & 0.784 & $0.71-0.85$ & - \\
$\quad$ New score & 0.699 & $0.61-0.79$ & 0.083 \\
Rockall score & 0.708 & $0.62-0.80$ & 0.049 \\
GBS full & & & \\
5 variables & 0.792 & $0.72-0.86$ & - \\
New score & 0.699 & $0.61-0.79$ & 0.046 \\
Rockall score & 0.708 & $0.62-0.80$ & 0.021 \\
GBS full & & & \\
\hline
\end{tabular}

GBS: Glasgow-Blatchford score.

hemostasis and epinephrine plus any second hemostasis were all effective methods for achieving hemostasis [9]. In our results, monotherapy was found to be independent risk factor for both rebleeding. Thus, "Monotherapy" was also set as one factor in our new scoring system. Finally, 4-variable and 5 -variable scoring systems were identified.

The 4-variable scoring system, which consists of 4 variables, namely, "HB $<85 \mathrm{~g} / \mathrm{L}$," "BUN $>9.5 \mathrm{mmol} / \mathrm{L}$," "Scr $>100 \mu \mathrm{mol} / \mathrm{L}$," and "Monotherapy", performed as well as RS and outperformed GBS significantly in predicting rebleeding. Moreover, the cut-off 2 for this scoring system provided very high sensitivity and good specificity in predicting rebleeding. If the intensive treatment was only performed on scores of 2, 3, and 4, almost all of high-risk patients could receive special care to decrease the risk of rebleeding while about $45 \%$ clinical resource could be saved. The 5-variable scoring systems, which comprise of 5 variables, "SBP $<90 \mathrm{mmHg}$," " $\mathrm{HB}<85 \mathrm{~g} / \mathrm{L}$," "BUN $>9.5 \mathrm{~m}$ $\mathrm{mol} / \mathrm{L}$," "Scr $>100 \mu \mathrm{mol} / \mathrm{L}$," and "Monotherapy", outperformed RS and GBS in predicting rebleeding. The cut-off 2 provided very high sensitivity in predicting rebleeding, but the specificity was lower than that of 4 -variable scoring system. In these 2 scoring systems, the patients with a score of zero could be normally managed as they were less likely to suffer rebleeding after endoscopic hemostasis.

As we know, the difficulty of calculating and distrust led to the factor that doctors were unwilling to use risk score the guidelines recommended for management of UGIB patients. Our simple score, no matter 4 variables or 5 variables were reliable, was easy-remembered and easy-calculated.

Despite the good performance of our new scoring systems, there were several limitations. One limitation was the small sample size. The small sample size makes it hard to draw a firm conclusion while many risk factors for clinical outcomes might be covered up. Moreover, the comparison of different endoscopic hemostasis methods such as thermal hemostasis versus multiple therapies combined and mechanical hemostasis versus multiple therapies combined was hard to achieve due to the small sample size. Another limitation is that our study is retrospective single-center clinical trial, which limits its reliability and generalizability potentially. 
In summary, $\mathrm{SBP}, \mathrm{HB}, \mathrm{BUN}, \mathrm{Scr}$, and endoscopic hemostasis methods were indicators for rebleeding in ANVUGIB patients with high-risk stigmata after endoscopic hemostasis. The 4-variable scoring systems we made were greater than GBS in predicting rebleeding significantly and 5-variable scoring systems we made outperformed RS and GBS in predicting rebleeding. However, larger multicenter prospective studies are needed to validate these conclusions and verify the new scoring systems thresholds that might be suitable for clinical decisions in the future.

\section{Conflicts of Interest}

The authors declare that there is no conflict of interest regarding the publication of this paper.

\section{Acknowledgments}

The current study was supported by the National Science Foundation of China (Grant no. 81370491), National Science Foundation of China (Grant no. 8157040827), and National Science Foundation of China (Grant no. 81502041).

\section{References}

[1] V. Jairath, B. C. Kahan, S. J. Stanworth et al., "Prevalence, management, and outcomes of patients with coagulopathy after acute nonvariceal upper gastrointestinal bleeding in the United Kingdom," Transfusion, vol. 53, no. 5, pp. 1069-1076, 2013.

[2] I. Khamaysi and I. M. Gralnek, "Acute upper gastrointestinal bleeding (UGIB) - initial evaluation and management," Best Practice \& Research Clinical Gastroenterology, vol. 27, no. 5, pp. 633-638, 2013.

[3] J. Y. Lau, J. Sung, C. Hill, C. Henderson, C. W. Howden, and D. C. Metz, "Systematic review of the epidemiology of complicated peptic ulcer disease: incidence, recurrence, risk factors and mortality," Digestion, vol. 84, no. 2, pp. 102-113, 2011.

[4] A. Lanas, L. Aabakken, J. Fonseca et al., "Variability in the management of nonvariceal upper gastrointestinal bleeding in Europe: an observational study," Advances in Therapy, vol. 29, no. 12, pp. 1026-1036, 2012.

[5] F. Kanwal, A. Barkun, I. M. Gralnek et al., "Measuring quality of care in patients with nonvariceal upper gastrointestinal hemorrhage: development of an explicit quality indicator set," The American Journal of Gastroenterology, vol. 105, no. 8, pp. 1710-1718, 2010.

[6] J. J. Sung, F. K. Chan, M. Chen et al., "Asia-Pacific Working Group consensus on non-variceal upper gastrointestinal bleeding," Gut, vol. 60, no. 9, pp. 1170-1177, 2011.

[7] L. Laine and D. M. Jensen, "Management of patients with ulcer bleeding," The American Journal of Gastroenterology, vol. 107, no. 3, pp. 345-360, 2012.

[8] I. M. Gralnek, J. M. Dumonceau, E. J. Kuipers et al., "Diagnosis and management of nonvariceal upper gastrointestinal hemorrhage: European Society of Gastrointestinal Endoscopy (ESGE) Guideline," Endoscopy, vol. 47, no. 10, pp. a1-46, 2015.

[9] M. Fujishiro, M. Iguchi, N. Kakushima et al., "Guidelines for endoscopic management of non-variceal upper gastrointestinal bleeding," Digestive Endoscopy, vol. 28, no. 4, pp. 363-378, 2016.
[10] T. A. Rockall, R. F. Logan, H. B. Devlin, and T. C. Northfield, "Risk assessment after acute upper gastrointestinal haemorrhage," Gut, vol. 38, no. 3, pp. 316-321, 1996.

[11] O. Blatchford, W. R. Murray, and M. Blatchford, "A risk score to predict need for treatment for uppergastrointestinal haemorrhage," The Lancet, vol. 356, no. 9238, pp. 1318-1321, 2000.

[12] E. R. Delong, D. M. Delong, and D. L. Clarkepearson, "Comparing the areas under two or more correlated receiver operating characteristic curves: a nonparametric approach," Biometrics, vol. 44, no. 3, pp. 837-845, 1988.

[13] N. de Groot, J. Bosman, P. Siersema, and M. van Oijen, "Prediction scores in gastrointestinal bleeding: a systematic review and quantitative appraisal," Endoscopy, vol. 44, no. 08, pp. 731-739, 2012.

[14] H. M. Yang, S. W. Jeon, J. T. Jung et al., "Comparison of scoring systems for nonvariceal upper gastrointestinal bleeding: a multicenter prospective cohort study," Journal of Gastroenterology and Hepatology, vol. 31, no. 1, pp. 119-125, 2016.

[15] A. N. Barkun, M. Martel, Y. Toubouti, E. Rahme, and M. Bardou, "Endoscopic hemostasis in peptic ulcer bleeding for patients with high-risk lesions: a series of meta-analyses," Gastrointestinal Endoscopy, vol. 69, no. 4, pp. 786-799, 2009.

[16] M. Horibe, T. Kaneko, N. Yokogawa et al., "A simple scoring system to assess the need for an endoscopic intervention in suspected upper gastrointestinal bleeding: a prospective cohort study," Digestive and Liver Disease, vol. 48, no. 10, pp. 1180-1186, 2016. 


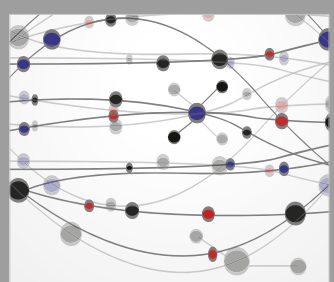

The Scientific World Journal
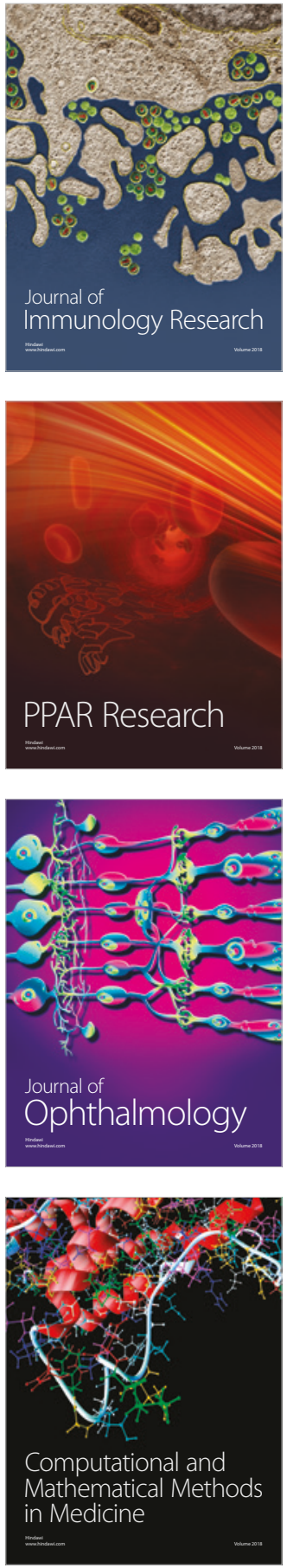

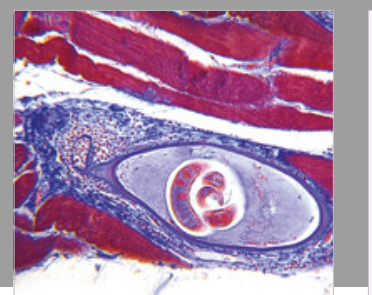

Gastroenterology Research and Practice

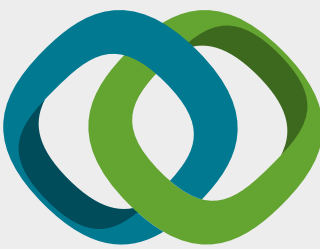

\section{Hindawi}

Submit your manuscripts at

www.hindawi.com
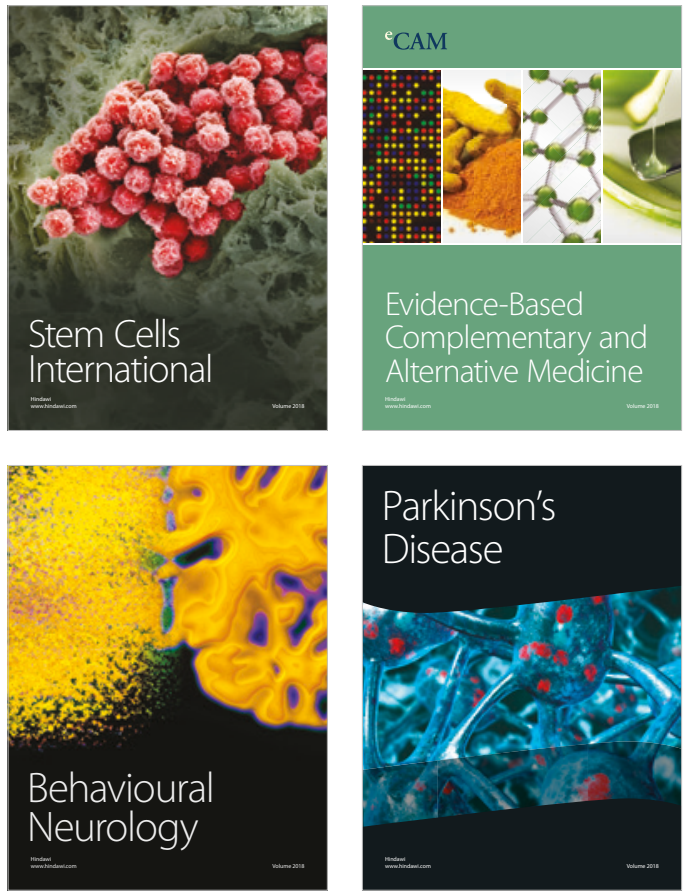

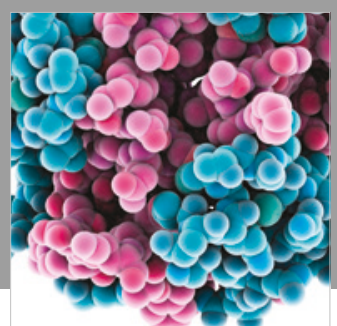

ournal of

Diabetes Research

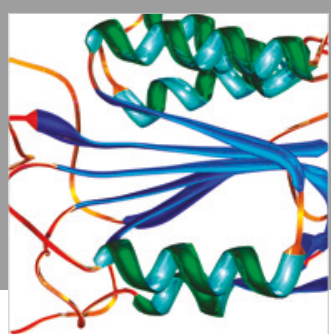

Disease Markers
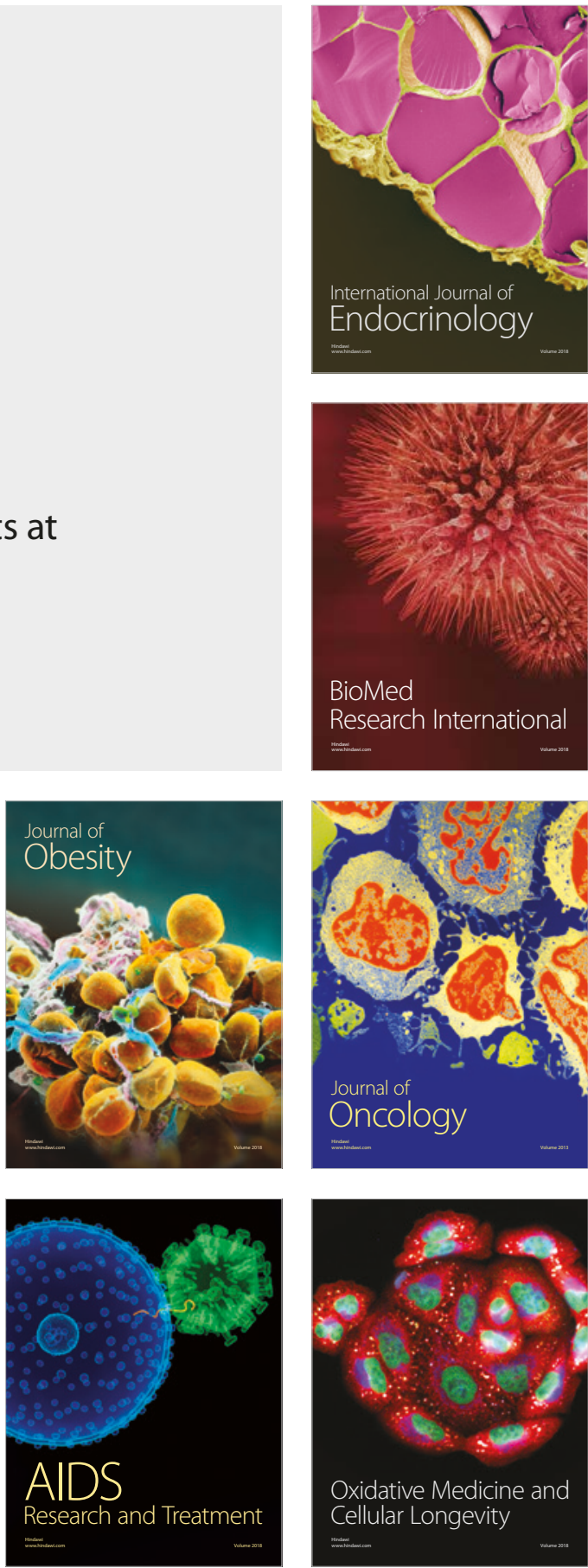